\title{
Esophageal sebaceous glands diagnosed after endoscopic mucosal resection
}

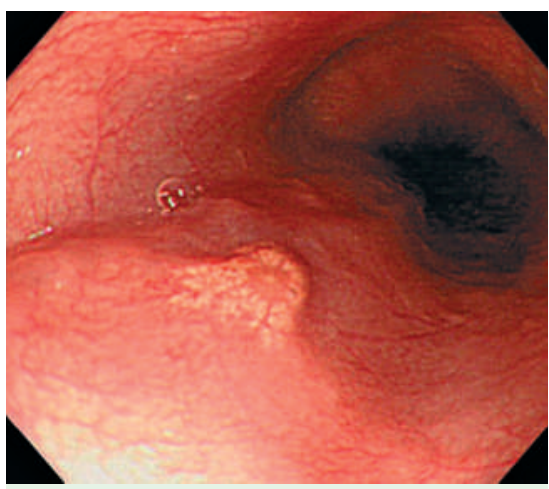

Figure 1 Esophagoscopy showing a yellow, oval, elevated lesion resembling gastric xanthoma.

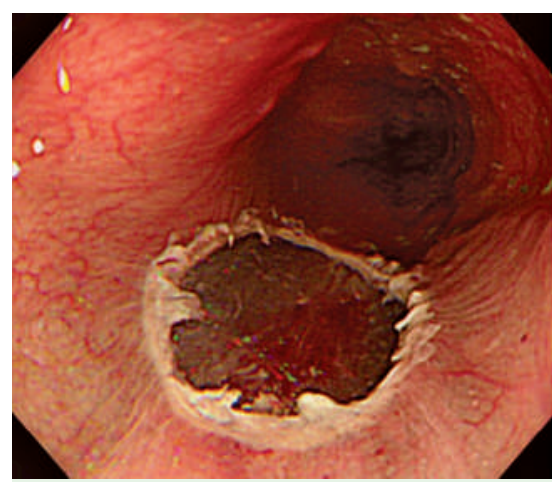

Figure 2 The endoscopic mucosal resection using the ligating device.

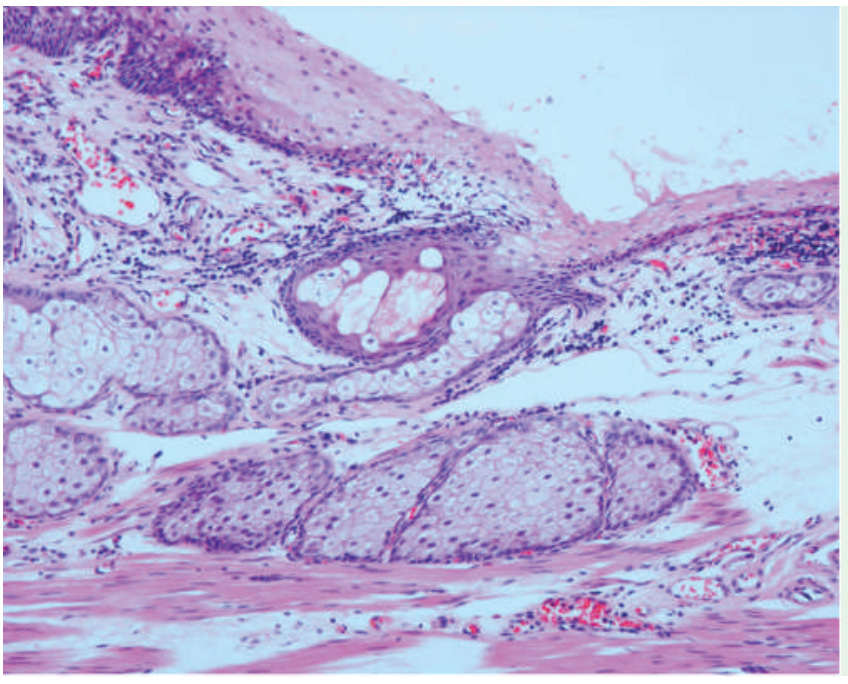

Figure 3 A small lobule of sebaceous glands was found to be embedded under the squamous epithelium of the esophageal mucosa (hematoxylin and eosin staining).
A 44-year-old woman with a duodenal ulcer underwent routine upper endoscopy; a yellow, oval, elevated lesion in the middle esophagus was found ( Figure 1 ). The lesion was slightly stained with Lugol iodine solution. Endoscopic ultrasound revealed that the hypoechoic solid tumor was limited to the esophageal mucosa and submucosa. Biopsy specimens with conventional forceps were noncontributory, showing normal covering squamous epithelia. To obtain sufficient tissue, we performed endoscopic mucosal resection using a ligating device (EMRL [1]).

We successfully resected the entire lesion without any complications ( $\bullet$ Figure 2 ). The size of the resected area was $7 \times 15 \mathrm{~mm}$, and that of the lesion was $5 \times 7 \mathrm{~mm}$. Histopathologically, a small lobule of sebaceous glands was found to be copy, the lesions were yellow, oval, and elevated, resembling gastric xanthomas. Being aware of the characteristics will allow this condition to be diagnosed $[4,5]$. In our case, however, conventional endoscopic biopsies were noncontributory, and we used EMRL for a definitive histopathological diagnosis. The lesion was safely and successfully resected with a clean margin.

Endoscopy_UCTN_Code_CCL_1AB_2AC_3AB

\section{T. Sekita ${ }^{1}$, S. Shikuwa ${ }^{1,2}$, H. Isomoto ${ }^{2}$,} N. Yamaguchi ${ }^{1}$, M. Ito ${ }^{3}$, S. Kohno ${ }^{1}$

Department of Internal Medicine, National Nagasaki Medical Center, Omura, Japan

2 Second Department of Internal Medicine, Nagasaki University School of Medicine, Nagasaki, Japan

Department of Pathology, National Nagasaki Medical Center, Omura, Japan

\section{References}

1 Suzuki Y, Hiraishi H, Kanke Ket al. Treatment of gastric tumors by endoscopic mucosal resection with a ligating device. Gastrointest Endosc 1999; 49: 192 - 199

2 Zak FG, Lawson W. Sebaceous glands in the esophagus. Arch Dermatol 1976; 112: $1153-1154$

3 Ramakrishnan T, Brinker JE. Ectopic sebaceous glands in the esophagus. Gastrointest Endosc 1978; 24: $293-294$

4 Hoshika K, Inoue S. Endoscopic detection of ectopic multiple minute sebaceous glands in the esophagus. Report of a case and review of the literature. Dig Dis Sci 1995; 40 : 287-290

5 Bertoni G, Sassatelli R, Nigrisoli E et al. Ectopic sebaceous glands in the esophagus: report of three new cases and review of the literature. Am J Gastroenterol 1994; 89: $1884-1887$

\section{Bibliography}

DOI 10.1055/s-2007-966546

Endoscopy 2007; 39: E161

(c) Georg Thieme Verlag KG Stuttgart · New York . ISSN 0013-726X

\section{Corresponding author}

\section{H. Isomoto, MD}

Second Department of Internal Medicine Nagasaki University School of Medicine

1-7-1 Sakamoto

Nagasaki 852-8501

Japan

Fax: +81-95-8497285

hajimei2002@yahoo.co.jp 